\title{
Electron Transport through Double Quantum Dots with Interdot Coulomb Repulsion
}

\author{
D. SzTenkiel And R. Świrkowicz
}

Faculty of Physics, Warsaw University of Technology

Koszykowa 75, 00-662 Warszawa, Poland

Electron transport through a system of two quantum dots connected in series is studied theoretically with the use of non-equilibrium Green function formalism based on the equation of motion method. Each dot is described by the one-level Anderson Hamiltonian and interdot Coulomb interactions in the form of the Hubbard-like term are taken into account. The electric current and occupation numbers are calculated with the use of two different approaches. The results of the methods are compared and discussed in detail. Strong asymmetry of $I-V$ characteristics with respect to bias voltage reversal are obtained when energy levels of the dots are not aligned.

PACS numbers: 73.23.-b, 73.21.La, 73.23.Hk

\section{Introduction}

Electron transport through double quantum dots (DQDs) attached to external electrodes is of current interest due to a possibility of using the structures in quantum computer hardware. Dots connected in series as well as in parallel configuration were investigated theoretically and experimentally [1-7]. $I-V$ characteristics obtained for such systems show a pronounced asymmetry with respect to a bias reversal $[3,6,7]$. Negative differential conductance was also observed. Recently the problem has been studied theoretically for a molecule attached to external leads. A diagrammatic technique for Hubbard operator Green functions (GFs) has been used $[8,9]$.

In this work we present a theoretical study of electronic transport through a DQD system based on a non-equilibrium GF formalism and use the equation of motion (EOM) method. The dots are treated as one-level Anderson impurities with interdot hopping processes described by the parameter $t_{\mathrm{C}}$ and interdot Coulomb repulsion. In dependence on the hopping strength the system can be treated as a two-dot system connected in series (a weak coupling regime) or as a molecule attached to external electrodes (a strong coupling regime). Two different mathematical approaches can be used to investigate the transport in these two 
regimes. When the hopping is very small in comparison to the coupling with the leads, $t_{\mathrm{C}} \ll \Gamma$, it is quite natural to treat DQD and external electrodes as a one system and to use an appropriate method to solve the problem. For example a procedure derived by Sun and Guo [10] can be used. On the other hand, in the regime of a strong interdot coupling $t_{\mathrm{C}} \gg \Gamma$ from physical point of view it is more justified to treat the system as a molecule effectively coupled to reservoirs and to diagonalize the DQD Hamiltonian [8, 9, 11-13].

In the present paper we consider the intermediate regime with $t_{\mathrm{C}}$ being of the same order as $\Gamma$. The current flowing through the system and the occupation numbers on the dots are calculated with the use of the two approaches mentioned above. First, we follow the procedure derived in Ref. [10] and then diagonalize the DQD Hamiltonian by means of a unitary transformation [13]. Results of both methods are compared and discussed for different values of hopping parameter from the intermediate regime. The calculated $I-V$ characteristics are very similar in both cases. Strong asymmetry with respect to bias reversal is obtained when energy levels in the dots are not aligned.

\section{Model}

We consider electron tunnelling through DQD attached to external leads. The system can be described by the following Hamiltonian: $H=H_{\mathrm{el}}+H_{\mathrm{DQD}}+H_{\mathrm{T}}$. Here, $H_{\mathrm{el}}=\sum_{k, \beta=\mathrm{L}, \mathrm{R}} \varepsilon_{k \beta} a_{k \beta}^{+} a_{k \beta}$ represents the Hamiltonian of the left and right electrodes, where $a_{k \beta}^{+}$and $a_{k \beta}$ are creation and annihilation operators of an electron in the electrode $\beta$ with the wave vector $k$ and energy $\varepsilon_{k \beta}$. When a bias voltage is applied the electrodes have different electrochemical potentials $\mu_{\mathrm{L}}=\mathrm{eV}$ and $\mu_{\mathrm{R}}=0$. The term describing DQD region is taken in the following form:

$$
H_{\mathrm{DQD}}=E_{\mathrm{L}} d_{\mathrm{L}}^{+} d_{\mathrm{L}}+E_{\mathrm{R}} d_{\mathrm{R}}^{+} d_{\mathrm{R}}+t_{\mathrm{C}}\left(d_{\mathrm{L}}^{+} d_{\mathrm{R}}+\text { h.c. }\right)+U d_{\mathrm{L}}^{+} d_{\mathrm{L}} d_{\mathrm{R}}^{+} d_{\mathrm{R}} .
$$

A single dot in the system is described by a one-level Anderson Hamiltonian with an infinite intradot Coulomb repulsion. Accordingly, only a single state on each dot is important and the double occupancy of the dot can be ignored. In the expression (1) $E_{\mathrm{L}}\left(E_{\mathrm{R}}\right)$ corresponds to the energy level of the left (right) dot and $d_{\mathrm{L}}\left(d_{\mathrm{R}}\right)$ is the appropriate annihilation operator. The hopping processes between the dots are described by the parameter $t_{\mathrm{C}}$. The interdot Coulomb repulsion between electrons is taken into account in the Hubbard form with the correlation parameter $U$.

The term $H_{\mathrm{T}}=\sum_{k, \beta=\mathrm{L}, \mathrm{R}}\left(t_{k \beta} a_{k \beta}^{+} d_{\beta}+\right.$ h.c. $)$ describes tunneling processes between the dots and the reservoirs. $t_{k \beta}$ represent here elements of the tunnel matrix. In the following we assume constant (independent of energy) coupling parameters $\Gamma_{\beta}(\omega)=2 \pi \sum_{k}\left|t_{k \beta}\right|^{2} \delta\left(\omega-\varepsilon_{k \beta}\right)=\Gamma_{\beta}$.

The current flowing through the system under the applied voltage is calculated according to formula derived by Jauho et al. [14]:

$$
I_{\beta}=\mathrm{i} \frac{2 e}{\hbar} \int \frac{\mathrm{d} \omega}{2 \pi} \Gamma_{\beta}\left[G_{\beta \beta}^{<}+f_{\beta}\left(G_{\beta \beta}^{\mathrm{r}}-G_{\beta \beta}^{\mathrm{a}}\right)\right],
$$


where $G_{\alpha \beta}^{i}(\omega)=\left\langle\left\langle d_{\alpha}, d_{\beta}^{+}\right\rangle\right\rangle_{\omega}^{i}$ are the Fourier transforms of the lesser, retarded and advanced GF for $i=<, \mathrm{r}$, a, respectively. Term $f_{\beta}$ corresponds to the Fermi-Dirac distribution function of the lead $\beta$. For a steady-state transport with constant coupling parameters $\Gamma_{\beta}$ the procedure derived by Sun and Guo [10] can be used. It allows to express the current in terms of average values $n_{\alpha \beta}=\left\langle d_{\alpha}^{+} d_{\beta}\right\rangle=-\mathrm{i} \int \frac{\omega}{2 \pi} G_{\beta \alpha}^{<}$ which can be calculated by solving a system of linear equations. It is important that in the steady state only retarded and advanced GFs appear in these equations. The procedure allows to omit any additional approximations involved in calculation of $G^{<}$(for details see [10]). The retarded and advanced GFs are calculated within the framework of the Hartree-Fock approximation with the use of EOM method. For DQD system GFs are represented by $2 \times 2$ matrices. In the present approach diagonal and non-diagonal terms are taken into account. The system of EOMs can be written in the matrix form which corresponds to the Dyson equation $\left(\hat{I}-\hat{g}_{0} \hat{\Sigma}\right) \hat{G}=\hat{g}_{0}$. The term $\hat{\Sigma}$ represents a self-energy of interacting system and $\hat{g}_{0}$ with elements $g_{0 \alpha \beta}=\delta_{\alpha \beta}\left(\omega-E_{\beta}\right)^{-1}$ is the GF of the uncoupled dot without Coulomb interactions. The calculated system of equations is solved in a self-consistent way and average values as well as the current are determined.

The second approach assumes that DQD system can be treated as a molecule and $H_{\mathrm{DQD}}$ can be diagonalized. The bonding and antibonding molecular states are given by the standard expressions [13] $\varepsilon_{i}=\frac{1}{2}\left[E_{\mathrm{R}}+E_{\mathrm{L}}+(-1)^{i} \sqrt{\Delta^{2}+4 t_{\mathrm{C}}^{2}}\right]$ with $\Delta=E_{\mathrm{R}}-E_{\mathrm{L}}$. In a presence of the bias voltage energy levels $E_{\mathrm{L}}$ and $E_{\mathrm{R}}$ are shifted in the following way: $E_{\mathrm{L}}=E_{\mathrm{L} 0}+\frac{2}{3} e V, E_{\mathrm{R}}=E_{\mathrm{R} 0}+\frac{1}{3} e V$, where $E_{\mathrm{L} 0}$ and $E_{\mathrm{R} 0}$ correspond to dot levels in the unbiased system. Then, the Hamiltonian describing the molecule connected to reservoirs can be written in the form [15]:

$$
H=H_{\mathrm{el}}+\sum_{i=1,2} \varepsilon_{i} c_{i}^{+} c_{i}+U c_{1}^{+} c_{1} c_{2}^{+} c_{2}+\sum_{k \beta=\mathrm{L}, \mathrm{R}, i=1,2}\left(T_{k \beta}^{i} a_{k \beta}^{+} c_{i}+\text { h.c. }\right),
$$

where $c_{i}^{+}\left(c_{i}\right)$ creates (annihilates) an electron in the molecular state $\varepsilon_{i}$. Matrix elements $T_{k \beta}^{i}$ describe tunneling processes between the molecule and the leads. They depend on hopping parameter $t_{\mathrm{C}}$ as well as on the applied voltage through the level positions $E_{\mathrm{L}}$ and $E_{\mathrm{R}}$. Hamiltonian (3) has the form identical to the one describing a magnetic dot attached to ferromagnetic leads when the molecular states $i=1,2$ with energies $\varepsilon_{i}$ are formally treated as corresponding to two different spin states [16]. Then, the current can be calculated in the HartreeFock approximation with the use of the procedure derived in [16]. When the DQD region is symmetrically coupled to the reservoirs with $\Gamma_{\mathrm{L}}=\Gamma_{\mathrm{R}}=\Gamma$ the system fully corresponds to the one consisting of magnetic quantum dot attached to ferromagnetic electrodes with antiparallel alignment of magnetic moments.

\section{Numerical results}

Electric current and occupation numbers are calculated numerically using both methods presented above. Parameters used in numerical calculations are 
measured in relative units with the coupling $\Gamma$ between DQD and one of the electrodes taken as the energy unit. We consider symmetrical case with $\Gamma_{\mathrm{L}}=$ $\Gamma_{\mathrm{R}}=\Gamma$. Calculations were performed for the following values of parameters: $k_{\mathrm{B}} T=\Gamma, U=20 \Gamma$. The hopping rate $t_{\mathrm{C}}$ was treated as a tunable parameter.

Let us consider first the case when energy levels of the two dots are aligned in the unbiased system $\left(E_{\mathrm{L} 0}=E_{\mathrm{R} 0}=4 \Gamma\right)$. The current and occupation numbers as functions of the bias voltage calculated with the use of both approaches described above are presented in Fig. 1. One can see that the results of both methods are well consistent. In the low voltage regime the current increases and it takes a maximal value at a certain voltage (Fig. 1a). Then it starts to decrease as energy levels $E_{\mathrm{L}}, E_{\mathrm{R}}$ are shifted by the bias voltage and they do not align. The current is fully symmetric with respect to the bias reversal. The occupation numbers of the left $n_{\mathrm{L}}$ and right $n_{\mathrm{R}}$ dots are very close to each other in the low voltage region where the current increases and displaces a peak (Fig. 1b). For higher values of $|e V|$ energy levels of the dots are strongly shifted and electrons start to accumulate on the dot close to the source. Mean number of electrons on this dot increases approaching unity. The other dot is almost empty. It strongly blocks the transport. According to the figure these processes are also fully symmetric.

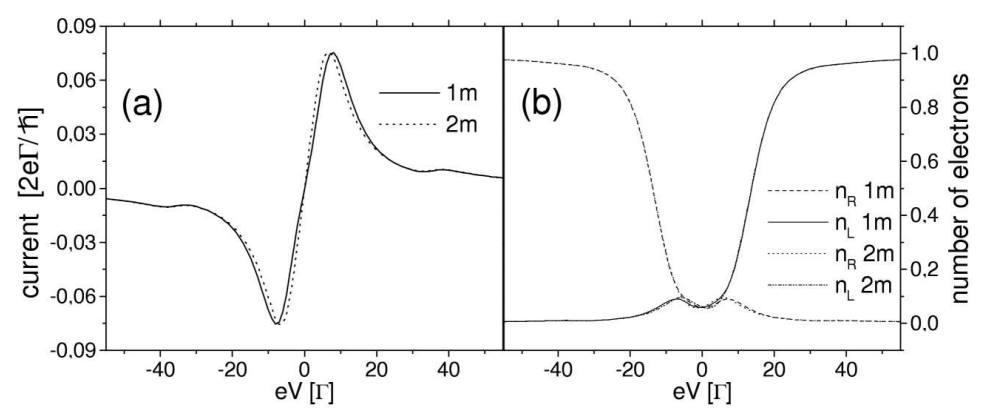

Fig. 1. (a) Electronic current and (b) occupation numbers in dependence on bias voltage calculated with the use of both approaches: first method $(1 \mathrm{~m})$ and second method $(2 \mathrm{~m})$. The relevant parameters are as follows: $E_{\mathrm{L} 0}=E_{\mathrm{R} 0}=4 \Gamma, U=20 \Gamma$, $k_{\mathrm{B}} T=\Gamma, t_{\mathrm{C}}=\Gamma$.

Next, we discuss the case when energy levels $E_{\mathrm{L} 0}, E_{\mathrm{R} 0}$ are strongly shifted. Electric current, differential conductance and occupation numbers of the left and right dots in dependence on the applied voltage are presented in Fig. 2 for various values of hopping rate $t_{\mathrm{C}}$. The left and right parts present results obtained with the use of the first and the second methods, respectively. The comparison shows that both approaches give very similar results, though some quantitative differences appear for a weak interdot coupling $t_{\mathrm{C}}=0.5 \Gamma$. When the diagonalization procedure is used in this case the current transmitted through molecular levels increases more rapidly and the differential conductance is somewhat higher 

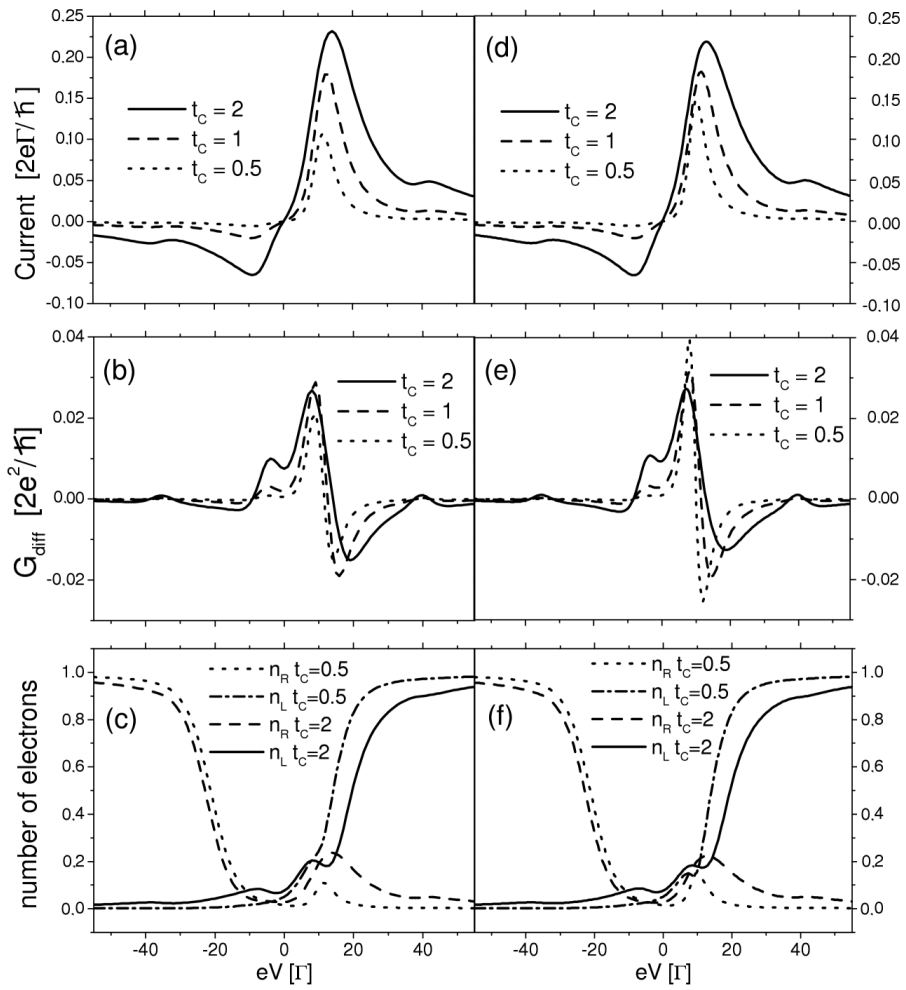

Fig. 2. Electronic current, differential conductance and occupation numbers as functions of bias voltage for different values of hopping rate. The parameters used in calculation are chosen as follows: $E_{\mathrm{L} 0}=4 \Gamma, E_{\mathrm{R} 0}=7 \Gamma, U=20 \Gamma, k_{\mathrm{B}} T=\Gamma$. The left and right parts present results obtained with the use of the first and second methods, respectively.

than the one calculated with the use of the first method (Fig. $2 \mathrm{~b}$ and e). It also leads to some minor differences in occupation numbers. However, the results are qualitatively consistent and the most important features of $I-V$ characteristics can be well seen in both cases. The calculated $I-V$ characteristics and differential conductance are strongly asymmetric with respect to the bias reversal. The asymmetry is much more pronounced for the weak interdot coupling $t_{\mathrm{C}}=0.5 \Gamma$. In this case the current shows a very well defined resonant peak for positive values of $e V$, whereas it is very small for all negative values of $e V$ (Fig. 2a and d). No peak can be seen in this range of voltages. The differential conductance is also very small and flat for $\mathrm{eV}<0$ (Fig. 2b and e). For higher values of $t_{\mathrm{C}}$ the resonant peak present in the current for positive values of $e V$ becomes much higher and wider showing that transmission through the system strongly increases. Moreover, a small peak corresponding to energy level shifted by $U$ can be noticed in the current and $G_{\text {diff }}$. It is important that for negative values of $e V$ a resonant 
peak starts to emerge and its intensity strongly increases with $t_{\mathrm{C}}$. The peak can be well seen in $I-V$ characteristics (Fig. 2a and d) as well as in differential conductance depicted in Fig. 2b and e. The results presented here are well consistent with the ones obtained by Fransson and Eriksson for DQD system with the use of diagrammatic technique [8].

Changes of occupation numbers of the left and right dots under the applied bias voltage are presented in Fig. $2 \mathrm{c}$ and $\mathrm{f}$ for two values of the hopping rate $t_{\mathrm{C}}=0.5 \Gamma$ and $t_{\mathrm{C}}=2 \Gamma$. Though for $t_{\mathrm{C}}=2 \Gamma$ some delocalization of electrons between the dots takes place, it is not very strong and we can still consider a mean number of electrons on each dot. Calculations show that delocalization takes place mainly in the region of low voltages where the strong transmission occurs and the current displays a well pronounced peak. For higher values of $|e V|$ electrons are strongly localized on the dot close to the source, whereas the other one is almost empty.

\section{References}

[1] A.K. Hüttel, S. Ludwig, H. Lorenz, K. Eber, J.P. Kotthaus, Phys. Rev. B 72, 081310 (2005).

[2] N. Fujita, F. Wakaya, Y. Yuba, K. Gamo, Physica E 7, 420 (2000).

[3] T. Junno, S. Carlsson, H.Q. Xu, L. Samuelson, A.O. Orlov, G.L. Snider, Appl. Phys. Lett. 80, 667 (2002).

[4] D. Sztenkiel, R. Świrkowicz, Acta Phys. Pol. A 108, 885 (2005).

[5] M.L.L. de Guevara, F. Claro, P.A. Orellana, Phys. Rev. B 67, 195335 (2003).

[6] W.G. van der Wiel, S. De Franceschi, T. Fujisawa, J.M. Elzerman, S. Tarucha, L.P. Kouwenhoven, Rev. Mod. Phys. 53, 1 (2003).

[7] K. Ishibashi, M. Suzuki, T. Ida, Y. Aoyagi, Appl. Phys. Lett. 79, 1864 (2001).

[8] J. Fransson, O. Eriksson, Phys. Rev. B 70, 085301 (2004).

[9] J. Fransson, Phys. Rev. B 69, 201304 (2004).

[10] Q. Sun, H. Guo, Phys. Rev. B 66, 155308 (2002).

[11] G. Chen, G. Klimeck, S. Datta, G.H. Chen, W.A. Goddard III, Phys. Rev. B 50, 8035 (1994).

[12] G. Klimeck, G.L. Chen, S. Datta, Phys. Rev. B 50, 2316 (1994).

[13] R. Ziegler, C. Bruder, H. Schoeller, Phys. Rev. B 62, 1961 (2000).

[14] A.-P. Jauho, N.S. Wingreen, Y. Meir, Phys. Rev. B 50, 5528 (1994).

[15] D. Sztenkiel, R. Świrkowicz, Acta Phys. Pol. A 105, 523 (2004).

[16] W. Rudziński, J. Barnas, R. Swirkowicz, M. Wilczynski, Phys. Rev. B 71, 205307 (2005). 